\title{
Perinatal Cannabis Exposures and Autism Spectrum Disorders
}

\author{
Ruba Sajdeya ${ }^{a, b}$ Joshua D. Brown ${ }^{a, c}$ Amie J. Goodin ${ }^{a, c}$ \\ ${ }^{a}$ Consortium for Medical Marijuana Clinical Outcomes Research, University of Florida, Gainesville, FL, USA; \\ ${ }^{\mathrm{b}}$ Department of Epidemiology, University of Florida, Gainesville, FL, USA; 'Department of Pharmaceutical Outcomes \\ \& Policy, Center for Drug Evaluation and Safety (CoDES), University of Florida, Gainesville, FL, USA
}

\section{Key Points}

- Observational studies to assess the effects of perinatal cannabis exposures on neurodevelopmental outcomes, such as autism spectrum disorder (ASD), on offspring should be interpreted cautiously. While studies may control for risk factors related to environmental, social, behavioral, and genetic factors, the risk for residual confounding (i.e., factors not controlled for that may be associated with both the exposure and the outcome) remains significant in these types of studies.

- The current evidence is not sufficient to conclude that perinatal cannabis exposure is causally contributing to ASD in offspring; however, clinicians should be advised that most authorities strongly recommend the discontinuation of cannabis use in patients who become pregnant and should remain discontinued throughout the pregnancy and lactation periods.

- Surveys in pregnant women show that 3-7\% may use cannabis during pregnancy and $>70 \%$ of these may use it specifically for nausea and vomiting symptoms. There is also evidence that legal medical and recreational dispensaries recommend it for these purposes. Clinicians and patients should adhere to evidence-based treatment recommendations, and clinicians in particular should be familiar with the potential harms of cannabis use in pregnancy and communicate these clearly with pregnant patients.

\section{Keywords}

Cannabis and cannabinoids · Prenatal cannabis exposure · In utero cannabis exposure $\cdot$ Neurodevelopmental outcomes - Autism spectrum disorders

According to the Substance Abuse and Mental Health Services Administration, 3-7\% of pregnant women in the USA report using marijuana during pregnancy [1]. Cannabis use during pregnancy has doubled since the early 2000 s, in tandem with general population use increases $[2,3]$. Several health organizations, including the Ameri-

karger@karger.com www.karger.com $/ \mathrm{mca}$

Karger $\stackrel{\text { ' }}{5}$
C 2021 The Author(s)

Published by S. Karger AG, Basel

This is an Open Access article licensed under the Creative Commons Attribution-NonCommercial-4.0 International License (CC BY-NC) (http://www.karger.com/Services/OpenAccessLicense), applicable to the online version of the article only. Usage and distribution for commercial purposes requires written permission. can College of Obstetricians and Gynecologists $[4,5]$ and the US Surgeon General [6], recommend against cannabis use during pregnancy, citing concerns related to the adverse effects of smoking as well as some evidence for impaired neurodevelopment in the offspring.

Delta-9-tetrahydrocannabinol (THC), the primary psychoactive constituent of cannabis, can cross the placenta and the fetal blood-brain barrier, causing disruptions in the endogenous cannabinoid signaling involved in embryonic neurodevelopment in the fetus $[7,8]$. A recent research letter, published in Nature [9], examined the association between prenatal maternal cannabis use 
and the risk of neurodevelopmental disorders in offspring, with autism spectrum disorder (ASD) as the primary outcome. The study used retrospective data from all live births from the provincial birth registry in Ontario, Canada, between April 2007 and March 2012. A total of 503,065 and 497,821 children were included for the ascertainment of neurodevelopmental outcomes and analysis at 18 months and 4 years of age, respectively, using linked data from provincial health administrative databases. Matching techniques were applied to control for potential confounders, including maternal age, parity, education level, preexisting maternal conditions, substance use, smoking, socioeconomic factors, and prenatal care. Gestational age at delivery and obstetric complications were further adjusted for in the statistical analysis. Using Cox proportional hazard regression models, the study found an association between prenatal maternal cannabis use and the incidence of ASD in children. The incidence of ASD was 4.0 per 1,000 person-years in the exposed group compared to 2.4 in the unexposed group, with a hazard ratio of 1.51 (95\% confidence interval 1.17-1.96) in the matched cohort after full adjustment.

This study is among the few to examine long-term outcomes related to prenatal cannabis exposure longitudinally using a large representative sample. Such studies are rare and highly needed in prenatal cannabis exposure research. Unsurprisingly, the study received widespread media coverage, given the timeliness of the topic as the legalization and liberalization of cannabis use, both medically and recreationally, continues [10]. In this commentary, we discuss some limitations of this and similar studies that warrant cautious interpretation of these findings.

ASD is a complex neurodevelopmental condition characterized by impairment in social interactions and communication, abnormal reactivity to sensory inputs, and restricted and repetitive behaviors [11]. No single etiology has been identified to cause ASD. Instead, ASD is thought to be caused by multiple genetic, biological, and environmental factors that may act collectively to cause abnormalities in the brain structure or function [12]. Multiple risk factors have been identified to increase the likelihood of developing an ASD in children. These factors include hereditary (genetic and epigenetic) predisposition, having a sibling with ASD, certain genetic or chromosomal conditions, such as fragile $\mathrm{X}$ syndrome, prenatal exposure to certain chemicals, air pollution, and prescription drugs, such as valproic acid and thalidomide, advanced parental age at conception, maternal obesity, diabetes, or immune disorders, extreme prematurity, very low birth weight, and oxygen deprivation to the fetus's brain during delivery (see Fig. 1) [12-14]. The distribution of these risk factors may be behind the difference of ASD risk between the exposure and comparison groups. However, accounting for such risk factors was absent, due to limited availability of these data within birth registry and administrative claims.

Cannabis use is also linked to other negative pregnancy outcomes that could be contributing to ASD risk. For example, cannabis use is associated with a higher risk of fetal low birth weight, [15] which in turn has been identified as a risk factor for ASD [14]. However, low birth weight as an intermediate step in the association between prenatal cannabis exposure and the risk of ASD was not assessed. In perinatal research, adjusting for intermediate variables is essential in determining if these variables are underlying drivers of the associations of interest [16].

There was no differentiation between recreational and medicinal cannabis use within the ASD study. However, it is important to know the type of cannabis use since medical cannabis use may be associated with underlying conditions that could also increase the risk of ASD. For example, cannabis use is approved for medical use as adjuvant treatment for epilepsy in many jurisdictions worldwide [17] and can be concurrently used with antiepileptic medications linked to ASD. Given the complexity of ASD etiology, deciphering the role of these interrelated risk factors' is challenging, and residual confounding is highly likely to have occurred in this case. The rigorous matching and adjustment techniques used in this study likely reduced possible biases, but they did not necessarily eliminate bias. Nevertheless, observational studies remain the most feasible study designs to assess effects of in utero cannabis exposure where randomization is not an ethical option.

Another limitation of the study was its inability to measure cannabis exposure. Data collected on cannabis exposure were limited on many levels, including the time of exposure, duration, frequency, dose, types of cannabis products, and administration routes. In utero exposure was defined as "any" exposure, and this exposure likely significantly varied between offspring in terms of dose, $\mathrm{THC} /$ cannabidiol ratios, frequency, and duration. Yet, quantifying cannabis exposure has been a major challenge for most research that relies on self-report for exposure quantification. Additionally, exposure was reported only at 11 weeks and 13 weeks of gestation, providing data on exposure during the first trimester of pregnancy, and data on continued cannabis exposure after birth (i.e., during breastfeeding where THC may be transmitted to the
68

Med Cannabis Cannabinoids 2021;4:67-71 DOI: $10.1159 / 000515871$
Sajdeya/Brown/Goodin 


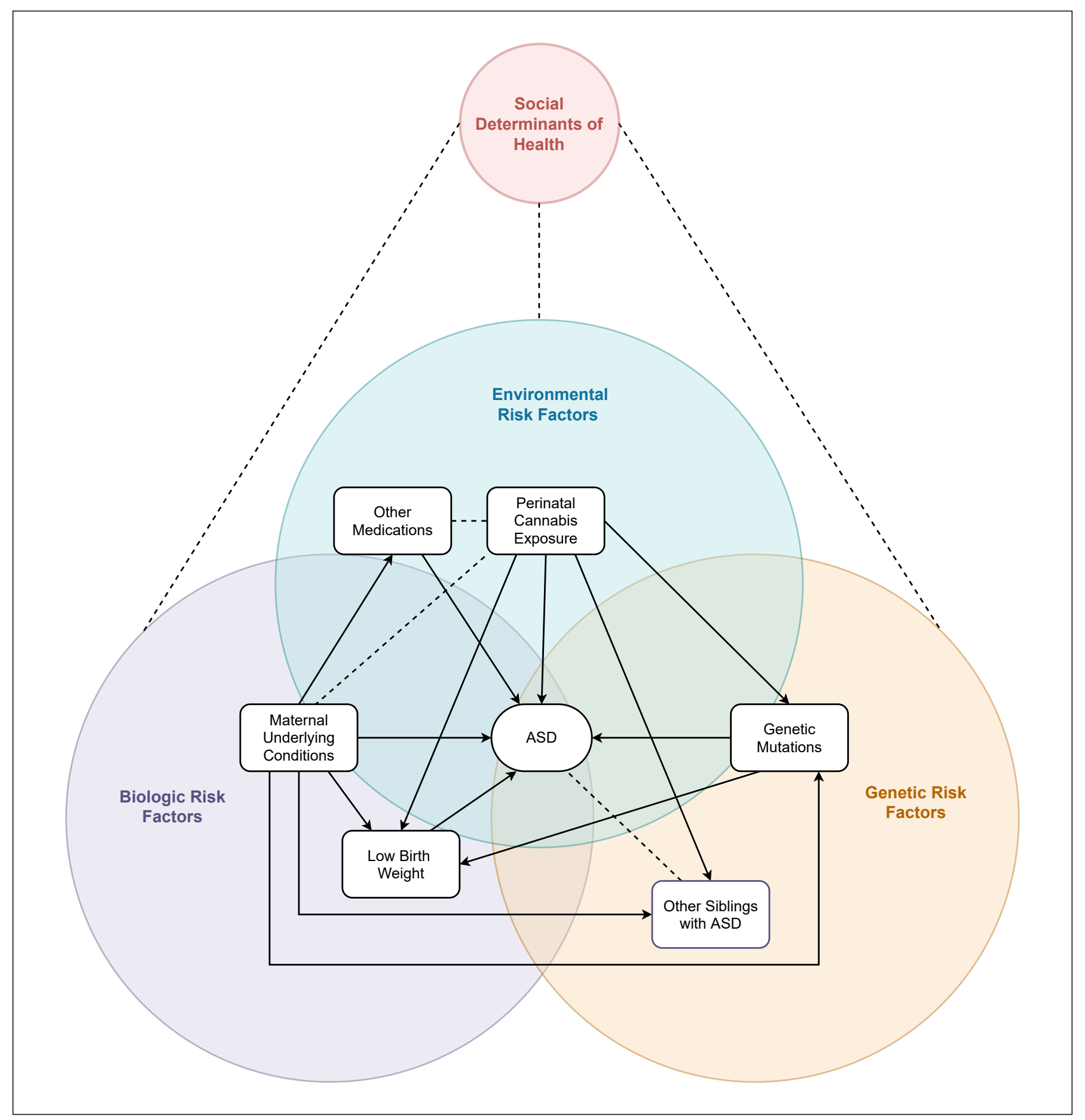

Fig. 1. Risk factors and exposures potentially associated with ASD. ASD, autism spectrum disorder.

offspring via breast milk) was also not available [4]. Evidence has also shown that the period preceding, during, and immediately after birth is critical in the development of ASD (Fig. 2) [18].

Evidence in Context
As such, a conclusion about a potential increased risk of ASD due to prenatal cannabis exposure cannot be made, as other important periods of potential hazardous exposure were not assessed. Nevertheless, expectant 


\section{Potential Stages and Mechanisms by Which Cannabis Exposure Could Interfere with Neurodevelopment and Increase the Risk of ASD}

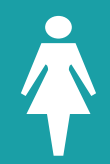

Preconception

Maternal hormonal,

immunological,

neurological, and

genetic (mutation)

changes

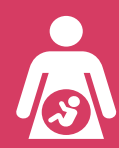

Early Prenatal

(0-13 weeks)

Interference with neural

tube formation, and

early endocannabinoid

system development

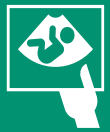

Late Prenatal

(14-40 weeks)

Interference with

endocannabinoid

system development

and increasing the risk

of low-birth weight (ASD

risk factor)

\section{Postnatal}

Exposure through

breastfeeding and

second-hand smoking

may interfere with CNS

development and

neurotransmitter

function

Fig. 2. Potential stages and mechanisms where cannabis exposure could interfere with neurodevelopment and increase the risk of ASD. ASD, autism spectrum disorders; CNS, central nervous system.

mothers should avoid using cannabis for recreational or medical purposes until further research is available given potential harms to infants beyond ASD [4-6]. Given most $(>70 \%)$ women who reported cannabis use during pregnancy used cannabis for nausea, vomiting, and poor appetite during pregnancy [19], patients and clinicians should adhere to evidence-based guidelines for treating nausea and vomiting in pregnancy [20]. There have been reports of both medical and recreational dispensaries recommending cannabis use in pregnancy for these symptoms, and so clinicians should be knowledgeable about the potential risks of cannabis in pregnancy in order to combat misinformation on its use in this scenario [21].

\section{Conclusion}

The authors acknowledged several of the outlined limitations of their study and recommended cautious interpretations of their findings. The evidence is not currently sufficient to conclude that perinatal cannabis use is a cause or a risk factor for the development of ASD. However, the Nature study does add to the evidence base as it establishes a framework for which other perinatal cannabis exposure research could be conducted, where long-term follow-up is essential for outcome ascertainment. More high-quality studies with longitudinal assessment of neurodevelopmental outcomes that simultaneously utilize a more comprehensive causal framework that can account for confounders, mediators, and other risk factors are highly needed. Such efforts should also attempt to measure cannabis exposure dose, duration, and frequency and cover other potential periods where exposures may potentially influence factors associated with ASD.

\section{Conflict of Interest Statement}

The authors have no conflicts of interest to declare.

\section{Funding Sources}

The authors are supported by State of Florida appropriations to the Consortium for Medical Marijuana Clinical Outcomes Research (mmjoutcomes.org).

\section{Author Contributions}

R.S. and A.G. conceptualized and drafted the work. R.S., J.B., and A.G. drafted and revised. All approved the final version. 


\section{Editor Note}

Evidence in context is part of the outreach effort of the Consortium for Medical Marijuana Clinical Outcomes Research to exam- ine and discuss implications of research into cannabis and cannabinoids for clinical practice, thus providing a translational approach to these studies to make clear, concise, and actionable evidence available for clinicians and patients.

\section{References}

1 Preventing the use of marijuana: focus on women and pregnancy $\mid$ publications and digital products. Available from: https://store. samhsa.gov/product/preventing-use-marijuana-focus-women-and-pregnancy. Accessed 2020 Nov 29.

2 Volkow ND, Han B, Compton WM, McCance-Katz EF. Self-reported medical and nonmedical cannabis use among pregnant women in the United States. JAMA. 2019 Jul 9;322(2):167-9.

3 Young-Wolff KC, Tucker LY, Alexeeff S, Armstrong MA, Conway A, Weisner C, et al. Trends in self-reported and biochemically tested Marijuana use among pregnant females in California from 2009-2016. JAMA. 2017; 318(24):2490-1.

4 Marijuana use during pregnancy and lactation. Available from: https://www.acog.org/ en/Clinical/Clinical Guidance/Committee Opinion/Articles/2017/10/Marijuana Use During Pregnancy and Lactation. Accessed 2020 Nov 30.

5 Committee opinion No. 722: marijuana use during pregnancy and lactation. Obstet Gynecol. 2017;130(4):e205.

6 Office of the Surgeon General AS for $\mathrm{H}$ (ASH). Surgeon General's Advisory: marijuana use \& the developing brain. HHS.gov. Published 2019 Aug 29. Available from: https:// www.hhs.gov/surgeongeneral/reports-andpublications/addiction-and-substance-misuse/advisory-on-marijuana-use-and-developing-brain/index.html. Accessed $2020 \mathrm{Nov}$ 30.

7 Brents LK. Marijuana, the endocannabinoid system and the female reproductive system. Yale J Biol Med. 2016;89(2):175-91.
8 Richardson KA, Hester AK, McLemore GL. Prenatal cannabis exposure: the "first hit" to the endocannabinoid system. Neurotoxicol Teratol. 2016;58:5-14.

9 Corsi DJ, Donelle J, Sucha E, Hawken S, Hsu H, El-Chaâr D, et al. Maternal cannabis use in pregnancy and child neurodevelopmental outcomes. Nat Med. 2020;26(10):1536-40.

10 State Medical Marijuana Laws. National Conference of State Legislature. Available from: https: //www.ncsl.org/research/health/statemedical-marijuana-laws.aspx. Accessed 2020 Nov 17.

11 What is autism spectrum disorder? Available from: https://www.psychiatry.org/patientsfamilies/autism/what-is-autism-spectrumdisorder. Accessed 2020 Nov 29.

12 Hyman SL, Levy SE, Myers SM; Council on children with disabilities Section on Developmental and Behavioral Pediatrics. Identification, evaluation, and management of children with autism spectrum disorder. Pediatrics. 2020;145(1):e20193447.

13 CDC. Basics About Autism Spectrum Disorder (ASD) | NCBDDD | CDC. Centers for Disease Control and Prevention. Published 2020 Mar 25. Available from: https://www. cdc.gov/ncbddd/autism/facts.html. Accessed 2020 Nov 29.

14 Autism. National Institute of Environmental Health Sciences. Available from: https:// www.niehs.nih.gov/health/topics/conditions/autism/index.cfm. Accessed 2020 Nov 29.
15 National Academies of Sciences E, Division $\mathrm{H}$ and $\mathrm{M}$, Practice $\mathrm{B}$ on $\mathrm{PH}$ and $\mathrm{PH}$, Agenda $\mathrm{C}$ on the HE of MAER and R. Prenatal, perinatal, and neonatal exposure to cannabis: National Academies Press; 2017. Available from: https:// www.ncbi.nlm.nih.gov/books/NBK425751/ Accessed 2020 Nov 30

16 Ananth CV, Schisterman EF. Confounding, causality and confusion: the role of intermediate variables in interpreting observational studies in obstetrics. Am J Obstet Gynecol. 2017;217(2):167-75.

17 Commissioner O of the FDA Approves first drug comprised of an active ingredient derived from marijuana to treat rare, severe forms of epilepsy. FDA. Published 2020 Mar 27. Available from: https://www.fda.gov/ news-events/press-announcements/fda-approves-first-drug-comprised-active-ingredient-derived-marijuana-treat-rare-severeforms. Accessed 2020 Nov 30.

18 Gardener H, Spiegelman D, Buka SL. Perinatal and neonatal risk factors for autism: a comprehensive meta-analysis. Pediatrics. 2011;128(2):344-55.

19 Brown QL, Sarvet AL, Shmulewitz D, Martins SS, Wall MM, Hasin DS. Trends in marijuana use among pregnant and nonpregnant reproductive-aged women, 2002-2014. JAMA. 2017;317(2):207-9.

20 Committee on Practice Bulletins-ObstetricsACOG practice bulletin No. 189: nausea and vomiting of pregnancy. Obstet Gynecol. 2018; 131(1):e15.

21 Dickson B, Mansfield C, Guiahi M, Allshouse AA, Borgelt LM, Sheeder J, et al. Recommendations from cannabis dispensaries about first-trimester cannabis use. Obstet Gynecol. 2018;131(6):1031-8. 